\title{
Ambiances
}

anbiances Environnement sensible, architecture et espace urbain Varia | 2013

\section{Flux, ambiances et ré-enchantement du monde}

Etude à partir de Malicroix d'Henri Bosco

Flux, atmospheres and the renchantment of the world. A case study based on Malicroix of Henri Bosco

\section{Olivier Labussière}

\section{(2) OpenEdition}

Journals

Édition électronique

URL : http://journals.openedition.org/ambiances/141

DOI : 10.4000/ambiances. 141

ISSN : 2266-839X

Éditeur :

Direction Générale des Patrimoines - DAPA - MCC, UMR 1563 - Ambiances Architectures Urbanités (AAU)

Référence électronique

Olivier Labussière, «Flux, ambiances et ré-enchantement du monde », Ambiances [En ligne], Varia, mis en ligne le 09 février 2013, consulté le 19 avril 2019. URL : http://journals.openedition.org/ ambiances/141; DOI : 10.4000/ambiances.141

Ce document a été généré automatiquement le 19 avril 2019

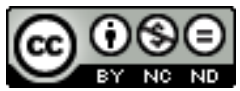

Ambiances is licensed under a Creative Commons Attribution-NonCommercial-NoDerivatives 4.0 International License. 


\title{
Flux, ambiances et ré-enchantement du monde
}

\author{
Etude à partir de Malicroix d'Henri Bosco \\ Flux, atmospheres and the renchantment of the world. A case study based on \\ Malicroix of Henri Bosco
}

Olivier Labussière

\section{Introduction}

1 La nature des ambiances est difficile à qualifier. Elle renvoie au registre de l'expérience mais une fois en ce domaine il est malaisé de savoir d'où surgissent les intensités perçues et comment elles reconfigurent la découverte d'un lieu.

Dans une étude consacrée aux ambiances urbaines, nous proposions de les caractériser par leur nature générative (Labussière, 2009). Une ambiance n'appartiendrait pas à une catégorie d'objets, pas plus qu'à un type de sujets disposant d'un goût spécifique. Elle procéderait d'une désorganisation progressive du perçu conduisant à un éclatement de la synthèse perceptive - ce pourquoi nous avançons l'idée du "moment d'ambiance ». Ceci fait de l'ambiance un ensemble élémentaire, difficilement descriptible mais hautement expressif qui invite à une découverte renouvelée du monde.

3 Cet article poursuit cette interrogation sur la nature des ambiances en prenant cette foisci pour piste de réflexion l'interaction entre le corps et le langage. Il y a là une tension qui, semble-t-il, reste à explorer. Bien souvent, l'ambiance paraît surgir au plus près du corps d'un rapport premier au monde qui ne doit rien au langage. Tout se passe comme si ce dernier intervenait en surplus, au travers de dispositifs méthodologiques lui conférant, enfin, un rôle dans la (re)connaissance du perçu (Thibaud, 2002; Grosjean \& Thibaud, 2001). Deux aspects semblent pourtant impliquer le langage dans les processus d'émergence des ambiances.

4 Tout d'abord, ne faut-il pas un long, patient et répété exercice de désorganisation de la langue pour parvenir au point où le corps s'ouvre à des perceptions proliférantes ${ }^{1}$, d'une 
multiplicité propre à générer une ambiance ? Comment parvenir au seuil de cette relation au monde où "tout le corps pense»? Comment épuiser la langue dans sa mission d'émissaire d'une conscience clairvoyante?

5 L'autre moment est celui où le corps s'étant ouvert à l'involontaire, il se trouve affecté par des perceptions de nature à recomposer la langue. Ces intensités perçues (sons, couleurs, odeurs...) opèrent une puissante remontée dans la langue au point qu'elles la pluralisent et la découvrent selon des contours qui ne sont plus ceux des rapports réglés du signifiant et du signifié. Quelle trajectoire le corps emprunte-t-il pour faire advenir cette langue renouvelée?

6 Pour aborder ces questions, nous nous saisissons d'un roman d'Henri Bosco (1888 - 1976), Malicroix. L'écriture d'Henri Bosco découvre une surprenante pensée concrète qui met à l'honneur les diverses manifestations d'un monde élémentaire (terre, vent, eau...) et produit de «formidables incubateurs de climats, d'ambiances, d'atmosphères » (Morzewski, 2002, p. 17).

7 Après une brève présentation du roman (i), nous étudions la figure de l'île (ii), centrale dans Malicroix, à partir d'un cadre conceptuel deleuzien. Pensée de flux et art de la dérive sont au cœur de cette approche qui suggère d'étudier les processus par lesquels le corps recompose le langage dans son ouverture aux éléments (iii). L'analyse de Malicroix est ensuite scandée par trois temps: la désorganisation du langage (iv), l'émergence de moments d'ambiance (v) et leur capacité à ré-enchanter un monde insulaire en déshérence (vi).

\section{Malicroix, une quête spirituelle?}

8 Henri Bosco réside au Maroc lorsqu'il prend sa retraite en 1945. Sa carrière d'enseignant de lettres classiques, menée entre autres en Italie et en France, s'achève au lycée Gouraud de Rabat. Au lendemain de la Seconde Guerre Mondiale, il est l'auteur de nombreux romans, pour certains primés ${ }^{2}$, dont il tire une notoriété suffisante pour arrêter son activité d'enseignement. Le roman abordé ici, Malicroix, paraît en 1948.

9 Le lecteur y découvre l'histoire d'un héritage : un jeune homme, Martial, élevé dans une famille simple et prospère, les Mégremut, hérite de son grand-oncle, Cornélius de Malicroix, une île au milieu du Rhône. Cornélius soumet cet héritage à deux conditions : un séjour de trois mois dans l'île et la descente du Rhône dans un bac à la dérive. Au cours de son séjour, Martial découvre par quelle tragédie le destin de son grand-oncle fut noué à l'île et au fleuve : alors que ce dernier partait en voyage de noces, son bac fut projeté sur un récif, causant la disparition de Delphine son aimé. Sur cette île sauvage, soumise aux pluies et aux vents, Martial a pour seule compagnie celle d'un vieux berger et de son chien, Baladran et Bréquillet, et la visite occasionnelle du notaire, Maître Dromiols, qui convoite l'héritage.

10 L'aventure initiatique, la solitude et le silence, l'accomplissement d'actes à forte charge symbolique sont les thèmes qui retiennent généralement l'attention. Ceci confère à l'expérience de Martial la valeur d'une quête spirituelle et tend à éclipser d'autres thèmes forts du roman, tels que le corps, le rapport aux éléments et au concret, qui sont cruciaux pour interroger les processus d'émergence des ambiances.

11 Ainsi, seul sur l'île, Martial apprivoiserait un « silence » (Michel, 1982) au sein duquel se découvrirait une «Présence» [sic]. L'aventure est spirituelle. L'île soumet le jeune 
homme à "une traversée de soi-même». Elle serait propice à la formation d'une intériorité. fleuve...). L'étude de M.-J. Salé (1988) sur la neige dans Malicroix fait exception. Elle indique que l'expérience insulaire progresse à travers des mondes successifs (ordinaire, poétique, spirituel). «Alliance de la blancheur et du silence ", la neige parle à l'âme, la purifie et vainc l'« être physique». Cette lecture dualise à l'excès le propos du roman (âme / corps) en ce qu'elle propose une hiérarchie des éléments trop définitive au regard du rôle de ceux-ci dans la narration: la neige salvatrice ferait ainsi contrepoint à la brutalité du fleuve et du vent.

nièle Henky (2002) soutient à son tour l'idée que l'île serait pour Martial un royaume du silence duquel la parole serait exilée. Martial passerait d'un silence subi à un silence voulu, autre illustration de cette quête spirituelle. Plus que d'autres pourtant, elle croise cette expérience du retrait avec l'idée d'une certaine volupté physique, d'une communion avec la nature. C'est là une voie intéressante qui n'est, selon nous, que partiellement explorée en ce que l'auteur associe l'expérience à une opération de synthèse et occulte ainsi la façon dont elle pourrait être informée par un milieu élémentaire dont elle n'a pas la maitrise. A ce niveau, il est intéressant de se tourner vers d'autres schémas de pensée susceptibles d'ouvrir l'expérience à des dimensions plus empiriques et vitales.

\section{Flux et dérive}

Une façon de procéder pour saisir le jeu des forces élémentaires et réfléchir sur l'émergence des ambiances dans leur rapport au langage, consiste à discuter la place accordée au milieu insulaire.

ernier a particulièrement été développé par Gilles Deleuze (2002), au travers du mythe de l'île déserte. André Pierre Colombat (1994) suggéra combien Bosco et Deleuze partageaient une préoccupation commune pour le vitalisme, sans pour autant relever que la figure de l'île pouvait en être la clef.

d'abord, un travail d'archive mit à jour des dessins d'Henri Bosco, dont un croquis de lîle qui donna lieu à la recherche de son modèle d'inspiration, l'île de la Barthelasse près d'Avignon (Girault, 1980), puis à une confrontation approfondie avec la géographie du roman (Girault, 2002). Une autre analyse (Michel, 1982) aborde le cadre insulaire selon une structure classique : un "élément solide isolé » entouré d'un "élément liquide isolant ». Elle décline cette structure au travers d'un réseau de symboles (espace clos / espace ouvert, fini / infini...) qui d'ailleurs réactive la quête spirituelle évoquée.

Dans Causes et raisons des îles désertes (Deleuze, 2002), Deleuze expose en quoi l'expérience de l'insularité est l'occasion d'un renouveau pour l'homme. En préambule, il postule que toute île est déserte, même habitée. Cette nature est invariable, c'est un état de séparation radicale justifié par l'espace incompressible qui l'entoure. "C'est l'homme qui se trouve séparé du monde en étant sur l'île » (Deleuze 2002, p. 12). L'île ne peut donc pas être ordonnée à partir de règles importées : elle débarrasse tout nouvel arrivant de sa gangue de normes et d'habitudes. Sans a priori, au seuil de l'origine, celui-ci entame un processus d'apprentissage tourné vers « les Eléments » (Deleuze 1972, p. 278). 

recommencement, elle assure la répétition du moment originel à partir duquel s'inventent de nouveaux devenirs. Et ceux qui viennent l'occuper se trouvent saisis par cette nature profonde. Ainsi, le naufragé « reprend et prolonge l'élan qui produisait celleci comme île déserte ; loin de le compromettre il le porte à sa perfection, à son comble " (Deleuze 2002, p. 13).

Comme nous avons déjà pu le montrer (Labussière, 2010), la spécificité de ce schéma deleuzien est de valoriser les qualités endogènes du milieu géographique en y trouvant toujours de nouvelles tendances à faire fructifier - cas particulièrement illustré par le Robinson de Michel Tournier (1972). Dans d'autres robinsonnades, lî̂le déserte est un espace-support conditionné par l'importation d'un modèle extérieur (Defoe, 2001) ou un espace-miroir réfléchissant un idéal de vie citadin (Giraudoux, 1997).

«Combat vivant de la terre et de l'eau » (Deleuze 2002, p. 11), voilà ce qu'est une île. Non une fraction terrestre que l'homme pourrait coloniser, mais un cosmos élémentaire dont le «mouvement» précède l'homme et trouve à travers ce dernier l'occasion de se redéployer. L'île est le lieu inépuisable d'apparition de la nouveauté.

Cette grille de lecture ne finalise pas - au sens du conditionnement par une fin - ce que peut être l'expérience insulaire. Le modèle est davantage celui d'un apprentissage consistant à expérimenter des modes de composition avec les éléments. Dans Malicroix, cet apprentissage est un art de la dérive devant conduire Martial, pour recevoir l'héritage, à laisser aller son bac «droit sur le Ranc [récif], au milieu des tourbillons " (Bosco 1948, p. 301), avant d'en « redresser la proue » au dernier moment.

Introduire dans le flux une déclinaison, produire une bifurcation d'où le monde pourra recommencer : cette dynamique au cœur de Malicroix invite à étudier la façon dont les ondulations de la langue participent à l'émergence des ambiances.

\section{Clinamen et turbulences}

Faire de l'île un havre de paix et du fleuve un flot menaçant, c'est restreindre la lecture de Malicroix à une dialectique de l'ancrage et de l'écoulement.

Le cadre deleuzien suggère une tout autre piste selon laquelle l'île est elle-même un flux, que l'homme ne fait que reprendre et prolonger. De ce point de vue, l'expérience insulaire est dans son entier une expérience de l'écoulement, un corps à corps continu avec les éléments. Ceci invite à étudier la recomposition permanente de Martial et de l'île au travers de mouvements élémentaires comme une introduction forte à l'épreuve finale, la descente du Rhône.

Dans l'île, Martial découvre au contact des éléments des occasions de dérive. C'est un processus spécifique qui ne peut être confondu avec une traversée du fleuve, ni confondu avec un abandon de soi, une communion spontanée avec la nature. La dérive est un apprentissage, un mode de navigation dans l'élémentaire, au travers des milieux, ce qui ne pourrait être saisi dès lors qu'on oppose les éléments entre eux ou le matériel au spirituel.

Pour dériver, il faut être pris dans un flux et faire advenir l'angle minimal qui produira une turbulence. Michel Serres (1977) offre une introduction magnifique à ce propos. Le monde est d'abord un écoulement laminaire, un flux, un flot. Pour que le monde 
(re)commence, il faut que ses lignes parallèles connaissent des fluxions, des écarts d'angles et que se forment des turbulences. «Le clinamen [mot latin signifiant déclinaison] est la plus petite condition concevable à la formation d'une turbulence " (Serres, 1977, p. 13).

Nous voici au seuil d'une interrogation renouvelée sur les processus d'émergence des ambiances dans leur rapport au langage. Notre questionnement initial suggère que la genèse d'une ambiance s'opère par un double fléchissement donné au langage : écart à la langue ordinaire / dominante et recomposition différentielle (ou «tourbillonnante»). Ainsi recomposé, le langage échapperait aux deux écueils du sens, le tout-laminaire et le tout-tourbillon : « il n'y a point de sens lorsque tout a le même sens. Il n'y a pas de sens lorsque tout est dans tous les sens »(Serres 1977, p. 179).

Pour sa part, Deleuze et Guattari nous invitent à considérer le langage en-deçà du sens auquel il est usuellement indexé. Echappant à l'ordre des significations, le langage libère « une matière vivante expressive qui parle pour elle-même et n'a plus besoin d'être formée » (Deleuze et Guattari 1975, p. 38). Ce peut être un son (piaulement, sifflement...), un mot (bégaiement, inflexion, répétition...) qui recomposent le langage « jusqu’à ce que ne subsistent plus que des intensités » (Deleuze \& Guattari, 1975, p. 35). Ce qui fait alors " sens ", c'est la métamorphose, l'évènement en tant qu'il enveloppe des états de choses hétérogènes (Deleuze, 1969). Le langage, pris dans une acception élargie, est donc aussi du côté des expérimentations du corps avec les éléments en tant qu'elles se manifestent au travers d'ensembles peu descriptibles mais hautement expressifs.

Cette mise au travail du langage participe de la génération de moments d'ambiance. Ce qui est exprimé dans l'ambiance procède à partir de déplacements au sein d'un milieu, ne renvoie pas à un sujet et produit un système hétérogène, « loin de l'équilibre » (Deleuze 2003, p. 185-186). Le langage n'est plus territorialisé, c'est-à-dire inscrit dans un ordre du discours, il devient tourbillonnant et manifeste un nouvel état médial et relationnel. Cette vitalité rappelle le passage du "langage-instrument » au «langage-réalité » décrit par Bachelard (1972, p. 10).

Aussi, il n'y aurait pas dans Malicroix des moments de silence qui seraient des situations de non-langage mais au contraire des «moments d'ambiance» qui traduiraient une déclinaison nouvelle du langage et une ouverture de Martial aux éléments.

\section{Langage laminaire et déviation élémentaire}

31 Malicroix est souvent présenté comme un roman initiatique dont l'enjeu serait la découverte d'une vie nouvelle dans le silence. Ce serait presque oublier que plusieurs voix en structurent le récit. Dans ce premier temps de l'analyse, nous étudions l'influence de la langue-Mégremut et de la langue de Dromiols sur l'organisation de la vie insulaire de Martial. L'une et l'autre expriment la puissance des ordres domestique et épistémique qui s'opposent à la découverte d'un milieu élémentaire.

\section{La langue-Mégremut, expression d'un ordre domestique}

Lorsque survient l'annonce de l'héritage, Martial vit chez les Mégremut, famille dont il est issu par son père. Cornélius de Malicroix est alors un ascendant méconnu, dont les Mégremut estiment que Martial ne porte "pas le nom» (p. 14) 3 . Dès les premières pages 
du roman, le langage est indexé à cet ordre domestique qui se manifeste à l'égard de Martial sur le mode du conseil, de l'autorité ou de l'ironie. Contre l'avis de tous, Martial part pour cette « terre à soucis» (p. 15).

Du moment où il monte dans la diligence jusqu'à son arrivée sur l'île, Martial n'a plus que des échanges ténus. Aux côtés de Baladran, personnage mystérieux qui lui sert de guide, le langage entre en mutation. Baladran s'exprime d'abord par des gestes et des signes (p.17). Sa parole est rare et n'intervient que sous une forme assourdie, au travers d'échanges interrompus (p. 20) ou par des réponses laconiques (p. 139). En guise de salut, Baladran grogne (p.157). Cette économie de la parole amorce un processus de déterritorialisation vis-à-vis de la langue-Mégremut, prévenante, explicite et conventionnelle.

La pauvreté de ce langage n'est qu'apparente. S'il informe peu Martial sur les intentions de son guide, il le conduit au seuil de réalités nouvelles.

J'étais en des lieux étrangers à ma vie naturelle. Les mots, les bruits, les silences, les objets mêmes, y parlaient un langage à eux, où je n'accédais pas (p. 35).

Sa tentation première est de réindexer ce langage flottant et inaccessible à un ordre domestique, celui de la Redousse, sa cabane, peuplée d'objets en apparence simples et bienveillants (p. 36). Il découvre progressivement que le mode d'existence des objets ne tient pas à leur utilité mais à une matérialité plus secrète, révélée par Baladran.

Je n'avais vu que des signes utiles là où Baladran saisissait la faïence écaillée de l'assiette, le grès grenu du pot, l'étain courbe du gobelet. Ses doigts leur donnaient l'existence [...] j'avais l'impression de découvrir un monde neuf, un monde coloré, solide, plein de son poids, entier (p. 212).

Avec Baladran apparaît une langue neuve appuyée sur la présence concrète des objets qui élargissent la perception qu'a Martial du domaine des corps et des affects.

La langue-Mégremut réapparaît à différentes occasions et inspire à Martial le souhait d'un retour à l'ordre domestique initial. La tentation du retour au même, présente à l'occasion de la réception de lettres de sa famille (p. 147) ou à l'approche de Noël (p. 182), manifeste le caractère vain de cette langue, dont les élans de tendresse échouent à saisir une vérité élémentaire.

Tous, attendris d'un rien, effrayés d'un rien, attristés d'un rien, fatigués d'un rien [...] avec nos maisons bien chauffées et bien fraîches, mi-soleil mi-ombre ; nos serres tièdes; nos lectures apaisantes [...] des hommes calmes et honnêtes, la bouche pleine de bon sens (p. 212).

L'île désorganise ce langage. Elle confère à Martial un regard décalé par rapport à cette «tribu la plus douce de la terre » (p. 149), dont les idées, les sentiments, les goûts coulent dans le même sens. En parallèle à l'épuisement de cet ordre domestique, se manifeste une autre voix, portée par Dormiols, le notaire.

\section{La langue de Dromiols, expression d'un ordre épistémique}

Si Martial parvient à se déprendre de la langue-Mégremut, bienveillant
affronte plus difficilement celle de Dromiols, faite d'éloquence et de raison.

La venue de Dromiols à la Redousse pour la lecture du testament prend l'allure d'un acte colonisateur. Survenant comme par effraction, il modifie autour de lui l'espace et le temps sur l'île: sa montre impose un temps mesuré (p. 73), il substitue au pain bis des mets raffinés (p. 78), son coucher est un véritable cérémonial avec récitation de vêpres en 
latin (p.102). Dromiols tire des malles qui l'accompagnent un ordre extérieur et catégorique.

Ce faisant, il contrarie l'apprentissage que Martial suivait auprès de Baladran dans une langue encore neuve et mystérieuse. Son éloquence et ses appels à la raison se déploient contre le monde élémentaire de l'île.

Les forces élémentaires vous écrasent : l'espace, l'eau, le vent. Ils vous assiègent, ils vous pressent, pénètrent en vous, et vous hantent, sans qu'à cette obsession vous puissiez opposer une résistance vraiment efficace (p. 86).

Dromiols adopte une position objectale qui réduit l'île à n'être qu'un point insignifiant de l'étendue. Son discours redistribue l'espace entre l'ici et le là-bas, entre une terre «pauvre » et « hostile » et les « terres riches et riantes » du pays natal de Martial.

Cette dénégation de l'île aboutit à ériger Cornélius de Malicroix en contre-exemple. Son isolement, ses mœurs tristes ont fait de lui « un possédé ». Il n'est pas cet oncle qui vécut en harmonie avec les éléments mais celui qui perdit « contact avec le monde » (p. 87), la preuve que « le pays n'est pas sain pour la raison» (p. 89).

Martial n'est pas dupe de cette éloquence. Mais il admire malgré lui cet homme bienparlant (p. 78) et ne trouve rien à lui opposer. Son mutisme, sa " parole simple et droite » (p. 90), son incapacité à masquer ses sentiments (p.69), le langage du notaire agit à son encontre comme un révélateur.

Dromiols déploie par son langage un véritable ordre épistémique qui vient structurer comme du dehors un monde primitif informe. Cet ordre détermine ce qui se donne à connaître et à penser. Il s'impose à Martial qui ne parvient pas à le contredire, comme si l'incapacité du jeune homme à manier le verbe rendait son corps plus débile et vulnérable face aux éléments. La ruse de Dromiols est double : elle baigne ce monde insulaire d'un verbe qui accentue son étrangeté et oblige Martial à le juger depuis l'ordre domestique des Mégremut.

Ce langage de raison s'inscrit aussi dans les choses et les corps : la lumière vacillante du chandelier de l'oncle Cornélius (p. 75), la pratique de la chiromancie sur Martial (p. 85), tout ceci contribue à brouiller le lien de descendance, le discours sur l'origine : «Pas un signe des Malicroix chez vous!»(p. 85), déclare satisfait Dromiols à Martial. En niant ainsi le milieu élémentaire de l'île, la langue du notaire produit de la déshérence, il rompt les liens entre le légataire et l'héritier.

47 En parti captif de cet ordre épistémique, Martial tente à de multiples reprises de raisonner sa condition, de compter les jours, de justifier son départ ou son maintien sur l'île (p.137). Cette oscillation entre le doute et la certitude ne produit pourtant pas de bifurcations majeures. Martial est l'illustration même de l'idée que la pensée n'est qu'un mode du corps et que plus que la pensée, c'est tout le corps qui pense (p.137). Ainsi, s'affirme en lui une profonde envie de rester dans l'île.

Mais d'une envie tenace, lourde, élémentaire. D'une envie qui ne voulait pas de justification ; car c'était une envie vitale, comme un grand besoin d'être [...] j'avais beau essayer d'expliquer ma conduite; mes arguments me semblaient risibles (p. 143).

Ces deux ordres de langage, domestique et épistémique, constituent des obstacles significatifs à la métamorphose de Martiale dans et avec l'île. Ils font de ce monde insulaire, au mieux une terre stérile où ne pourra jamais être reproduit l'idéal du jardin et au pire une île livrée aux éléments où tout heurte la norme et la raison. L'une plaide pour la continuité de la filiation sociale, l'autre pour la rupture de l'intermédiation des 
éléments entre les individus. En cela, ces deux langues sont laminaires. Elles réclament le retour au même et font d'un désir de dérive, de répétition du moment générateur de l'origine, une déviation condamnable.

En parallèle, Henri Bosco met le corps au travail dans sa capacité à déplacer le langage, à le déterritorialiser pour l'inscrire dans des aventures involontaires: l'extraordinaire matérialité des choses, la vitalité procurée par les éléments sont des indices d'une bifurcation à venir de Martial avec l'île.

\section{Formes tourbillonnaires et émergence des ambiances}

La métamorphose de Martial ne s'opère pas en une fois. C'est peut être ce qu'échoue à saisir une lecture de Malicroix focalisée sur une approche strictement symbolique. Par exemple, à survaloriser l'épreuve finale, celle où Martial manœuvre avec succès le bac sur le Rhône, le risque est de faire tenir tout le récit sur l'acte de bravoure du jeune héros.

51 Notre grille de lecture suggère davantage que Martial apprend sur l'île, au contact des flux élémentaires, un art de la dérive. Cette approche permet d'interroger la question des ambiances en liant à travers l'étude des étapes de leur émergence, la double perspective du corps et du langage. Notre étude porte sur deux passages du roman et examine trois étapes clefs de l'ouverture de Martial au milieu insulaire : un état d'errance, l'émergence d'une langue tourbillonnante, la formation d'un corps élémentaire.

\section{« Désormais j'étais fait de vent »}

Ce passage se situe dans le premier tiers du roman (chap. 3, "La Redousse», p. 123 - 132). Martial séjourne sur l'île depuis peu. Après avoir investi La Redousse, Dromiols s'en est allé laissant le jeune homme dans une solitude profonde.

Bien que seul, Martial n'éprouve ni «mélancolie», ni «tristesse» (p. 123). Il ne soupire pas après son pays natal et se trouve au contraire surpris «par le sentiment de la grandeur » (p. 123). La surprise est de taille car le notaire tenait par son langage l'île en proie à un destin néfaste.

Martial découvre un monde en marche: "l'immensité des eaux", "la majesté du fleuve ", « la montée des nuages» (p. 123). Ce «monde démesuré » (p.123) vient peupler sa solitude et ouvre le jeune homme au «sentiment de l'amplitude inspiré du dehors par la nature » (p. 123). L'état d'abandon se mue en éveil. Capté par les puissances primitives, il chemine le long du fleuve, erre sur des sentiers boueux, puis retourne à sa cabane. Martial ne perd pas conscience mais les limites de son âme se dilatent tandis que s'ouvre « un lieu indéfinissable, qui n'était ni en moi, ni hors de moi » (p. 125).

«Un évènement se formait au sein de ce repos, si dense, et on en pressentait la grandeur imminente » (p. 128). Le vent se lève. C'est de lui que va naître un langage nouveau. Sa rumeur génère un concert de voix : il fait entendre sa plainte, "ulule », « crie ", « rugit ", " aboie », "meugle ", "brame ", «barrit »... "La voix impérieuse du Vent-Maitre " (p. 130). Tout devient vent. L'île est prise dans un gigantesque flux élémentaire. Mais à la différence du fleuve, ce flux n'est pas laminaire. C'est ce qui singularise son langage. Henri Bosco identifie le vent à un mouvement en spirale: spirales ascensionnelles, tourbillon, tournoiement, ouragan. 
Entre l'ouragan et moi-même toute limite s'abîmait, et bientôt je devins un morceau volant de l'espace, à travers lequel tournoyait un faible sentiment de l'être universel (p. 131) moment encore obscurément liée au fleuve. Elle introduit Martial à un nouveau vagabondage. L'espace perd sa dimension d'étendue pour n'être plus que mouvement.

Je n'étais plus qu'une onde, une onde humaine qui vibrait au passage de la neige et qui devenait neige, et qui voltigeait (p. 186) s'éparpillent en permanence. A l'inverse, le fleuve court « de sa source à la mer, d'une seule coulée » (p. 186). Il n'a qu'un seul sens alors que la neige se dissémine dans l'espace. Cette conscience soudaine de l'écoulement unique du fleuve provoque chez Martial un mouvement de terreur.

Alors qu'il s'enfuit sa course produit un mouvement différentiel. Il réalise des rotations involontaires qui le ramènent, coup sur coup, trois fois au fleuve. De la répétition émerge une langue neuve, fruit de la rencontre entre les éléments dans et par la turbulence. Cette forme tourbillonnaire le fait rentrer dans «un monde assourdi » (p.189), composé "d'une seule onde immense»(p.190), et d'accéder à un autre fleuve, stellaire et étincelant. Le corps battu par la neige, Martial apprend à composer avec l'élémentaire : il 
progresse, par son mouvement erratique, du fleuve terrestre au flux laminaire vers les cieux tourbillonnants.

\section{Déshérence, ambiance et ré-enchantement du monde}

64 l'issue du plus sévère exercice de dépersonnalisation, qu'il acquiert son véritable nom propre »(Deleuze \& Guattari 1980, p. 51). Ce sont ces moments d'ambiance qui confèrent à Martial «un nom de cette terre $»^{4}$, le nom des Malicroix. Percevoir et nommer se retrouvent au cœur d'un même processus de décentrement et de ré-enchantement.

70 Arc-bouté sur sa rame, Martial descend le fleuve: son corps donne en même temps l'ultime et la première déclinaison à cet écoulement laminaire, dans ce tourbillon reprend le commencement du monde. 


\section{BIBLIOGRAPHIE}

Bachelard, Gaston. 1972. La poétique de l'espace. Paris : Presses Universitaires de France.

Bosco, Henri. 1948. Malicroix. Paris : Gallimard, (Folio)

Colombat, André-Pierre. 1994. Malicroix et le vitalisme français de Bosco à Deleuze. The French

Review, vol. 67, n 5, p. 803-812.

Defoe, Daniel. 2001 [1719]. Robinson Crusoé. Paris : Gallimard.

Deleuze, Gilles. 1969. Logique du sens. Paris : Les Editions de Minuit. Vingt-sixième série, du langage, p 212-216.

Deleuze, Gilles (postfacier). 1972. Michel Tournier et le monde sans Autrui. In : Michel Tournier. Vendredi ou les limbes du Pacifique. Paris : Gallimard.

Deleuze, Gilles. 2002. Causes et raisons des îles désertes. In : David Lapoujade (ed.). L'île déserte et autres textes. Textes et entretiens 1953-1974. Paris : Les Éditions de Minuit. p. 12-19

Deleuze, Gilles. 2003. Lettre à Uno sur le langage. In : Deux régimes de fous. Textes et entretiens 1975-1995. Paris : Les Éditions de Minuit. p 185-186.

Deleuze, Gilles \& Guattari, Félix. 1975. Kafka. Pour une littérature mineure. Paris : Les Editions de Minuit.

Deleuze, Gilles \& Guattari, Félix 1980. Capitalisme et schizophrénie. Mille plateaux. Paris : Les Editions de Minuit.

Giraudoux, Jean. 1997 [1921]. Suzanne et le Pacifique. Paris: Emile-Paul.

Girault, Claude. 1980. Propos sur Malicroix. Cahiers de l'amitié Henri Bosco, n¹9/20, p. 81-93.

Girault, Claude. 2002. Malicroix, l'île imaginaire. Roman 20-50, n 33, p. 24-36. Numéro consacré à «Henri Bosco, Malicroix et Un rameau de la nuit ».

Grosjean, Michèle \& Thibaud, Jean-Paul. 2001. L'espace urbain en méthodes. Marseille : Parenthèses.

Henky, Danièle. 2002. Malicroix, retraite au pays du souffle. Roman 20-50, n³3, p. 37-48. Numéro consacré à « Henri Bosco, Malicroix et Un rameau de la nuit ».

Labussière, Olivier. 2009. Éléments pour une symptomatologie des ambiances urbaines. Articulo revue de sciences humaines, Hors-série 2, Esthétiques et pratiques des paysages urbains. Disponible http://articulo.revues.org/1153 (consulté le 24 janvier 2011).

Labussière, Olivier. 2010. Optimisation, organisation de l'espace et pensée de l'émergence. La piste esthétique chez Gilles Deleuze. In : Actes du colloque Géopoint 2008, p. 79-84.

Michel, Jacqueline. 1982. Ecrire les îles : Henri Bosco, Jean Grenier. Cahiers Henri Bosco, n²2, p. 44-59.

Morzewski, Christian. 2002. Avez-vous lu Bosco ?, Roman 20-50, n 33, p. 11-24, Numéro consacré à «Henri Bosco, Malicroix et Un rameau de la nuit ».

Salé, M.-J. 1988. La neige comme élément naturel, poétique et initiatique dans Malicroix. Cahiers Henri Bosco, n 28, p. 226-238. 
Serres, Michel. 1977. La naissance de la physique dans le texte de Lucrèce. Fleuves et turbulences. Paris : Les Editions de Minuit.

Thibaud, Jean-Paul. 2002. L'horizon des ambiances urbaines. Communications, n 73, p. 185-201.

Tournier, Michel. 1972 [1967]. Vendredi ou les limbes du Pacifique. Paris : Gallimard.

\section{NOTES}

1. Cette intuition est fondée sur l'étude des cheminements de Marcel Proust dans Venise (Labussière, 2009).

2. L'âne Culotte (1937) et Le Mas Théotime (1945) sont récompensés par le prix Renaudot.

3. De façon à guider le lecteur dans la découverte du roman d'Henri Bosco, nous proposons de nombreuses références de pages, toutes issues de la même édition (Bosco, 1948). Ces références sont le plus souvent indicatives de passages plutôt que d'extraits du texte.

4. Ceci est le titre du sixième chapitre du roman qui en compte huit.

\section{RÉSUMÉS}

Cet article interroge l'idée selon laquelle une ambiance surgit d'un rapport premier au monde et ne doit rien au langage qui serait l'outil d'une (re)connaissance. L'analyse est développée à partir d'un roman d'Henri Bosco, Malicroix. Dans ce roman, le cadre insulaire joue un rôle phare, pris en tension entre le langage de l'Autre (ordres domestique et épistémique) et les manifestations d'un milieu élémentaire. Tirant parti d'un cadre deleuzien, l'analyse montre comment une double inflexion donnée au langage reconfigure l'expérience du milieu dans le sens d'un corps à corps plus étroit avec les éléments, propice à l'émergence de moments d'ambiance dotés d'une expressivité propre. Auteur d'une langue concrète et vitale, Henri Bosco confère à ces moments d'ambiances, et donc au milieu dans lequel ils trouvent leurs appuis, la capacité de ré-enchanter un monde insulaire en déshérence.

This article questions the idea that atmospheres might emerge from the perceptions of the body and might not be linked in any case to the language. The analysis is based on a novel by Henri Bosco: Malicroix. This novel offers a narrative whose island setting is under the dual and concurrent influences of the language of the Other (domestic and epistemic orders) and of an elementary world. Thanks to a deleuzian conceptual framework ("desert island"), the analysis puts under the light how a double inflection given to the language resizes the experience of the milieu in the sense of a broader embodiment with the elements, suitable for the emergence of moments of atmosphere. Author of a materialist and a vitalist language, Henri Bosco gives to these moments of atmosphere, and through them to the milieu, the ability to re-enchant a fallen island's world. 
INDEX

Keywords : atmosphere, language, body, milieu, Gilles Deleuze, Henri Bosco, island

Mots-clés : ambiance, langage, corps, milieu, Gilles Deleuze, Henri Bosco, île

\section{AUTEUR}

OLIVIER LABUSSIÈRE

Institut de Géographie Alpine

Laboratoire PACTE

Maître de conférences en géographie et aménagement

olivier.labussiere@ujf-grenoble.fr 